\title{
A Rede Social Facebook em contexto de investigação e colaboração mediadas uma experiência Luso-Brasileira
}

\section{The social network "Facebook" under a mediated research and colaboration framework a Luso-Brazilian experience}

\author{
Elvira Rodrigues*, Joaquim Escola**
}

*Doutoranda em Ciências da Educação na Universidade de Trás os Montes e Alto Douro (UTAD); ** Docente da Universidade de Trás-os-Montes e Alto Douro, UTAD, Escola de Ciências Sociais e Humanas

\begin{abstract}
Resumo
Nesta comunicação apresentamos o relato de utilização de um grupo secreto na rede social facebook em contexto educativo, no ensino superior. Esta experiência decorreu no âmbito do seminário "Questões Avançadas de Comunicação Educativa" que integra o curso de doutoramento em Ciências da Educação da UTAD.

Este estudo exploratório inclui um enquadramento teórico à metodologia adotada no Ambiente Virtual de Aprendizagem e apresenta e discute os resultados obtidos a partir dos inquéritos de diagnose e de satisfação realizados, conjugados com os dados emergentes da observação direta e participante.

Palabras chave: Facebook; colaboração; AVA;
\end{abstract} aprendizagem mediada.

\section{Abstract}

In this article we present the usage report of a secret facebook group within an educational framework, in higher education. This experience took place in the seminar "Advanced Issues of Educational Communication" integrating the doctoral program in UTAD Educational Sciences.

This exploratory study It includes a theoretical framework of the methods used to develop a learning virtual environment in the social network, and we present and discuss the results obtained from the diagnosis and satisfaction survey coupled with the follow-on data from direct and participant observation.

Keywords: Facebook; collaboration; learning virtual environments; cooperative learning

\section{Introdução}

No âmbito do seminário "Questões Avançadas de Comunicação Educativa" que integra o curso de doutoramento em Ciências da Educação da UTAD desenvolvemos um grupo secreto na rede social facebook.

$\mathrm{Na}$ construção do AVA (Ambiente Virtual de Aprendizagem) partimos de um modelo que integra 5 dimensões: social e de partilha, negociação, compromisso mútuo e cooperação; colaboração e construção de conhecimento; liderança e moderação em amb ientes online e dimensão de construção de identidade em ambientes online, conjugado com as 4 fases de development research [pesquisa no desenvolvimento, muito usada em tecnologia educativa], para o desenvolvimento de um AVA e construção do conhecimento, como u ma elaboração de possibilidades para a criação e otimização do nosso grupo secreto na rede social facebook, entendido como u ma possível COP online, por considerarmos ser aquele que melhor respondia aos objetivos delineados para este estudo exploratório.

\section{Investigar no Século XXI: desafios da [rede]criatividade}

As novas técnicas e metodologias de aprendizagem e de formação, de que o e-learning e o b-learning são exemplo, associadas à partilha em comunidades de prática, são apenas alguns dos desafios que se colocam aos professores nesta segunda década do século XXI.

Perante esta avalancha de mudanças desafiadoras, torna-se cada vez mais necessário abrandar a marcha, perder tempo para ganhar tempo, conscientes de que a inovação tecnológica deverá ser um apoio às práticas pedagógicas assentes em modelos inovadores, nas tecnologias que os sustentam e nas teorias e modelos pedagógicos que os fundamentam. $\mathrm{O}$ construtivismo constitui o enfoque teórico que inspira a maioria das grandes e mais prometedoras inovações do ponto de vista do ensino-aprendizagem nas escolas.

$\mathrm{Na}$ atualidade, os acrónimos TIC, TICE e REA, Tecnologias de Informação e Co mun icação, Tecnologias de Informação e Comunicação aplicadas à Educação e Recursos Educativos Abertos, já se vulgarizaram e acentuaram a discussão sobre como ensinar e aprender em ambientes virtuais respondendo aos desafios do ciberespaço, globalização e interculturalidade (Escola, 2013:155) validando a aprendizagem não formal e informal.

As práticas de colegialidade, através da mútua supervisão e construção de saber interpares, as sumem-se como fundamentais nesta "Escola [ainda] Web 2.0", a qual assenta na partilha, colaboração, comunicação, confiança, inteligência coletiva e também na premissa de que inovação e utilização dos recursos tecnológicos

*Professora da Escola Secundária Augusto Gomes; Formadora do CFAE_Matosinhos, colaboradora do Centro de Estudos de Desenvolvimento Humano da UCP; ** Membro integrado do IF da Universidade do Porto.

* elvirarodrigues@esag-edu.net** jescola@utad.pt; www.utad.pt

Selección y peer-review bajo responsabilidad del Grupo de Investigación G000422-GIPDAE, Universidade da Coruña, España. 
exige uma metodologia de trabalho eficaz e um projeto pedagógico sustentado.

As facilidades de interação e de publicação online, sem que para isso sejam necessárias competências técnicas, habitualmente reservadas aos especialistas, alavancou novo(s) significado(s) à part ilha; à articu lação em tempo real entre os cibernautas, à "cidadania digital". Em simu ltâneo, novas palavras vieram engrossar o glossário da "gíria cibernaútica" no nosso quotidiano, como: comentar; googlar; mailar; "estar no face"..., para citarmos apenas algumas das mais utilizadas. Nesta segunda década do século XXI o grande desafio que, continua a colocar-se aos docentes, é o de apostarem numa aprendizagem colaborativa e provocatória da sua "rede criatividade" e da criatividade em rede dos seus alunos.

A coaprendizagem e a coinvestigação são práticas, cada vez mais, emergentes nesta segunda década do século XXI na ótica do que alguns já apelidam de aprendizagem e investigação criativa (Horizon Report Europe, 2014:1). Assim, cerca de onze anos após a criação do movimento REA, vivemos agora perante um "processo aberto" (Okada,2013:22), em que o conhecimento para além de rapidamente disseminado também pretende ser "des-e reconstruido-coletivamente (Idem, 2013:13).

\section{Método}

Para aferir as possíveis potencialidades de utilização de uma rede social em contexto de investigação, no âmbito de um seminário num curso de $3^{\circ}$ ciclo, doutoramento, enquanto modelo de aprendizagem mediada pela tecnologia, definimos três objetivos norteadores desta experiência, a saber:

- Aferir a importância da rede social facebook enquanto possível instrumento de construção colaborativa de conhecimento.

- Inferir o perfil de utilização do espaço virtual dos doutorandos envolvidos na utilização do grupo secreto.

- Compreender a influência do perfil dos dinamizadores e do feedback atempado e assertivo na motivação e participação dos colegas.

Uma revisão bibliográfica e sitográfica em torno da utilização das redes sociais em contexto escolar, no ato de ensino, e em contexto de investigação, revelou-se muito útil como ponto de partida para a emergência e criação deste grupo secreto luso brasileiro mediado pela rede social facebook.

Analisamos os modelos de aprendizagem construtivistas em plataformas digitais e, embora, já alguns autores que nos antecederam como Minhoto e Meirinhos (2011:27), apontem o modelo de colaboração de Murphy (2004) como aquele que é passível de ser utilizado para a colaboração assíncrona online em comunidades colaborativas e de co construção do conhecimento, consideramo-lo redutor pelo facto de não efetuar qualquer referência a um dinamizador do grupo. Neste modelo, o desenvolvimento da colaboração opera-se num processo contínuo ao longo de seis etapas que vão evoluindo para um processo colaborativo: presença social; perspetivas de articulação individual; apropriação ou reflexão sobre as perspetivas dos outros; co construção de perspetivas e significados; articulação através de objetivos e propósitos compartilhados e produção compartilhada de tarefas e produtos. A presença social permite a coesão do grupo e fomenta a interação co mo condição impo rtante, mas não suficiente para a colaboração. Assim, optamos pelo modelo desenvolvido em 2009 por Pinto (Pinto, 2009) e que se baseia nos modelos de Henri (1992), Gunawardena et al. (1997) e Garrison et al. (2000), com elementos da proposta defendida por Wenger (1998) como mentor da definição e caracterização de COP (Comunidade de Práticas), e ainda da proposta de Dias (2008), que apresenta a moderação e a mediação colaborativa nas comunidades de aprendizagem e intenta construir um modelo de análise qualitativa das interacções específico para COP online (modelo de Miranda-Pinto, 2009). Esta proposta integra 5 dimensões: social e de partilha, negociação, empenhamento mútuo e cooperação; colaboração e construção de conhecimento; liderança e moderação em ambientes online $e$ dimensão de construção de identidade em ambientes online. Este emerge da necessidade da sua autora tentar ajustar e adaptar os modelos existentes ao projeto de uma COP online que se encontrava a des envolver no terreno. Nesta sugestão as interações nos espaços (fóruns) de discussão e no chat da plataforma são objeto de uma análise qualitativa, tentando inferir, por um lado, a interação de cada participante e, por outro, identificar o cic lo de vida de uma COP online (Quadro 1). Procuramos adaptá-lo à nossa realidade específica - um grupo secreto no facebook - que acabou por funcionar enquanto espaço de interação e partilha entre sete doutorandos, dois portugueses e cinco brasileiros: seis professores e uma enfermeira.

Quadro 1: Sistematização do modelo de Miranda-Pinto adaptado ao grupo secreto

\begin{tabular}{ll}
\hline Dimensão Social e de & *Presença como \\
Partilha & Observador-Participante e \\
& como explorador. \\
& *Partilha de experiências. \\
& *Corroborar comentários de um \\
& ou mais participantes. \\
& *Questionar e Responder para \\
& clarificar detalhes de alguma \\
& participação. \\
\hline Dimensão de Negociação, & *Identificação de áreas de \\
Empenhamento Mútuo e & interesse entre os participantes. \\
Cooperação & esclarecimento dos significados \\
(interatividade cognitiva) & dos diversos termos utilizados. \\
& *Proposta e negociação sobre \\
& novas áreas de debate. \\
& * Cooperação na realização de \\
& atividades entre os \\
& participantes. \\
& * Empenhamento mútuo de \\
& práticas diversificadas. \\
&
\end{tabular}




\begin{tabular}{ll}
\hline Dimensão de Colaboração e & *Partilha de informação, \\
Construção de & argumentação e integração de \\
Conhecimento & novos contributos. \\
(interatividade & *Debate sobre a informação \\
metacognitiva) & partilhada e estabelecimento de \\
& um consenso. \\
& *Reflexão crítica dos \\
& participantes e construção \\
& partilhada do conhecimento. \\
\hline Dimensão de Liderança e & *Identificação de Líderes e \\
Moderação em Ambientes & Moderadores. \\
online & *Estratégias de Liderança e \\
(fatores de sustentabilidade) & Moderação. \\
& *Evidência discursiva de \\
& orientação explícita. \\
\hline Dimensão de Construção & *Perceção da presença \\
de Identidade em & cognitiva. \\
Ambientes online & *Perceção da presença social. \\
\hline
\end{tabular}

Fonte: Miranda-Pinto (2009).

\section{Participantes}

Os participantes neste grupo secreto são sete doutorandos, dois portugueses e cinco brasileiros: cinco professores universitários no Brasil, umdos quais exerce cumulativamente advocacia, uma professora do $3^{\circ}$ ciclo e ensino secundário e uma enfermeira pediatra, em Portugal. Todos os elementos deste grupo Luso Brasileiro vivem em cidades diferentes. A inscrição no doutoramento em ciências da educação na Universidade de Trás-os-Montes e Alto Douro e a frequência do seminário de "Questões Avançadas de Comunicação Educativa" é um dos pontos de referência entre todos eles.

As questões logísticas e de enquadramento começaram a ser preparadas no início da lecionação do seminário "Questões Avançadas de Co municação Educativa", após um inquérito de diagnose inicial e de uma reflexão operacionalizada em torno das conclusões emergentes dos dados de um inquérito que permitiu caraterizar os estilos de utilização do espaço virtual destes sete doutorandos, 4 do sexo femin ino e 3 do se xo masculino. As suas habilitações literárias iniciais são bastante heterogéneas, a saber: bacharelato em engenharia elétrica; direito; dire ito e matemática; história; sistemas de informação; bacharel em comunicação social com habilitação em publicidade e propaganda e enfermagem. Estes estudantes de $3^{\circ}$ ciclo em ciências da educação possuem mestrado em direito; administração de empresas; administração; ensino de ciências da saúde e do meio a mbiente; história moderna; engenharia elétrica e computação e ensino de ciências da saúde e do meio ambiente. Contudo, e para além disso, a sua formação adicional ainda se reparte por: especialização em planeamento estratégico de negócios; direito tributário; contabilidade pública e responsabilidade fiscal; docência para ensino superior; redes de computadores; especialização em docência do ensino superior; especialização em engenharia de segurança do trabalho e administração de marketing; direito tributário; contabilidade pública; mestrado em ciências da educação, especialização em supervisão pedagógica; livre docência em direito e especialidade em saúde infantil e pediatra. Os recursos e potencialidades das Tecnologias Educativas (TE), de uma forma mais ou menos explícita, estão presentes em todos os pré projetos que se propuseram desenvolver no âmbito do curso de doutoramento em que se encontram inscritos.

\section{Procedimentos e instrumentos metodológicos}

Para a recolha de dados optamos pela elaboração de um inquérito por questionário realizado no início, como diagnose, triangulado com notas de campo durante as aulas e participação online.

A parte do inquérito no que diz respeito à identificação do estilo de utilização do espaço virtual pelos inquiridos foi traduzido e adaptado para google drive do inquérito inserto em Barros (2009:73,74). Assim, este foi apresentado com as quatro possibilidades (participativo; seleção e pesquisa; estrutura e planeamento e ação concreta), separado por "páginas" e em que os inquiridos tinham de identificar as respostas verdadeiras em cada uma das quatro secções.

Após definirmos os conteúdos a abordar e a estrutura genérica do nosso grupo secreto, enquanto possível ambiente virtual, continuamos o processo de pesquisa, leitura e sistematização em torno da criação de um A VA.

Para a sua estruturação, como já mencionamos, optamos pelas 4 fases de development research [pesquisa no desenvolvimento, muito usada em tecnologia educativa], apontadas por Richey e Van Den Akker e mencionadas por Lencastre (2012:49) para o desenvolvimento de um AVA e construção do conhecimento acerca da sua implementação, a saber:

a. Investigação Preliminar: diagnose das competências TIC dos doutorandos e identificação do seu estilo de utilização do espaço virtual.

De seguida, selecionamos e sequenciamos as atividades a realizar e os materiais a partilhar, numa escala gradativa de dificuldade e de "criação de dependência viciante" face ao grupo secreto, nomeadamente através do recurso aos comentários a posts e a um feedback (mensagens) personalizado, assertivo e com recurso ao "reforço positivo", facultado num intervalo de tempo muito curto. Procurou-se, igualmente, criar condições para a discussão, interação e proatividade online. $\mathrm{O}$ recurso a imagens apelativas e com mensagens atrativas e assertivas, por nós selecionadas e renovadas frequentemente, foram, a par com uma navegação síncrona, algumas das estratégias utilizadas.

b. Sustentação teórica e recursos a exemplos de boas práticas: nesta segunda etapa, procuramos reforçar a nossa sustentação teórica e analisar exemplos de boas práticas online, beneficiando da nossa experiência de participação em vários grupos de investigação e construção de conhecimento partilhadas.

c. Teste empírico: durante este terceiro momento, o ma is demorado, fomos testando e avaliando soluções à medida que preparamos a articulação dos conteúdos a postar e dos aspetos multiméd ia. Foi, ta mbém, a altura de rever a expressão escrita e apurar algumas questões técnicas.

d. Avaliação das diferentes fases do desenvolvimento do design da avaliação e da implementação: por fim, 
procedemos à anotação das alterações efetuadas e realizamos printscreens do processo evolutivo do nosso grupo ao longo dos seis meses de duração do seminário. A monitorização foi constante e sistemática.

Para a análise dos dados optamos pela estatística descritiva (análise quantitativa) e pela análise de conteúdo das categorias emergentes da questão abertas do inquérito de diagnose inicial e das notas de campo.

\section{Apresentação e discussão dos resultados}

O estilo predominante no global dos nossos inquiridos é o estilo de seleção e pesquisa no espaço virtual, com $56 \%$, logo seguido do estilo participativo, co m $25 \%$, não sendo significativos os percentuais obtidos nos estilos relativos à estrutura e planeamento e ação concreta no espaço virtual.

Apenas um dos elementos, que se depreende ser a profissional de saúde, respondeu negativamente à questão sobre a utilização de plataformas digitais no des empenho da sua atividade profissional. Nesse âmbito, destacam-se três plataformas: moodle; facebook e webeduca. A totalidade dos inquiridos afirma ser capaz de projetar um curso online, estruturar as atividades de aprendizagem, os planos de aula e os desafios, selecionando as ferramentas mais adequadas, bem como utilizam as redes sociais, para de forma privada, trabalharem com os seus pares. Contudo, e não obstante estas respostas, apenas $28,6 \%$ recorre ao trabalho colaborativo online com muita frequência, enquanto que $57,1 \%$ nunca dinamizou um A VA (A mbiente Virtual de Aprendizagem) - 42,9\% apenas utiliza o grupo secreto sobre o qual recai este estudo como plataforma mediada de partilha e co construção de conhecimento. Os restantes $57,1 \%$ integram grupos de investigação, onde a co construção de conhecimento de forma presencial e mediada, é uma constante e ocorrer essencialmente através do facebook; whatsapp, google, drive e partilha de alguns materiais através da dropbox.

Os dados provenientes das respostas às questões abertas do questionário de diagnose inicial encontravam-se em "bruto" exig indo procedimentos de: registo, análise, interpretação e explicitação.

Numa primeira etapa, efetuamos uma "leitura atenta e ativa" (A mado, 2013:311) das respostas a essas questões, simplificando os dados através da identificação de unidades de sentido num recorte e diferenciação vertical para posteriormente procedermos a um reagrupamento e comparação horizontal (Amado, 2013: 315 e 323). Obtivemos três categorias emergentes, categorias $a$ posteriori, as quais interpretamos de acordo com o quadro de referentes abaixo elencado (Quadro 2).

Quadro 2: Quadro de referentes das categorias emergentes do corpus investigativo

\begin{tabular}{ll}
\hline Facilidade & $\begin{array}{l}\text { Facilidade no acesso e visualização, } \\
\text { nomedamente através de um smartphone. }\end{array}$ \\
\hline Simplicidade & $\begin{array}{l}\text { Simplicidade na visualização e download } \\
\text { dos materiais, trabalhos e desafios } \\
\text { propostos. }\end{array}$ \\
\hline Interação & $\begin{array}{l}\text { Rapidez e fluidez na interação } \\
\text { doutorandos/orientador e doutorandos entre }\end{array}$
\end{tabular}

si. Possibilidade de respostas/comentários curtos com recurso a um feedback atempado e assertivo por parte do orientador, do colega dinamizador do grupo e dos colegas entre si.

Fonte: Questões abertas do Inquérito de satisfação final.

A rapidez de comunicação e interação entre os diversos intervenientes sobressaem na categoria "interação" conjugadas com a facilidade e simplicidade inerentes à comunicação mediada através de um grupo secreto no facebook, enquanto plaforma digital de partilha e co cons trução de conhecimento.

As conclusões de utilização deste grupo secreto de forma sistemática e regular ao longo de meio ano evidencia que o recurso, de forma regular e sistemática ao grupo, ocorre essencialmente em momentos de resolução de necessidades mu ito específicas, ou procura de materiais, e/ou notícias partilhados por alguns colegas em geral, e pela colega dinamizadora, de uma forma intensiva e cuidada. Os downloads ma is efetuados pelos doutorandos assumiram-se co mo tendo interesse direto e, ma is ou menos imediato, co m os projetos de investigação de cada um dos membros que integra o grupo. Assim, para a sua manutenção muito contribuiu a insistência na contínua partilha de conteúdos e desafios por parte da colega que assumiu o papel de dinamização deste grupo secreto e o facto de, na sua otimização, ter tentado operacionalizar aquilo que convencionou apelidar de coaching online ou peer coaching com recurso a um feedback atempado, assertivo e com recurso a imagens apelativas. A presença síncrona e diálogos personalizados, nomeadamente através de skype, com especial incidência com alguns dos colegas do Brasil, assumiu-se como um importante reforço ao papel desempenhado pelo grupo.

Num exercício de meta reflexão, e aplicando a este grupo secreto o tetra desenvolvido por Marhall MacLuhan (1988) na obra "A Lei dos Media", podemos afirmar que este grupo amplificou as possibilidades e potencialidades de interação; transfor mou os espaços de intervenção; recuperou a proximidade entre os seus membros e tornou obsoleta a "desculpa" da distância geográfica numa trilogia de oportunidade; desafio e sedução para os diferentes intervenientes deste espaço de investigação e colaboração mediadas.

\section{Considerações finais}

Com esta experiência monitorizada e análise dos resultados obtidos, procuramos apresentar os dados emergentes da utilização de um grupo secreto na rede social facebook com 7 doutorandos do Curso de Doutoramento em Ciências da Educação, no âmbito do seminário de "Questões Avançadas de Comunicação Educativa".

Este trabalho prospetivo deixa em aberto questões que poderão ser objeto de estudo em futuros trabalhos de investigação. Por exemplo, em nossa opinião, conclusões interessantes poderiam emergir da triangulação destes dados com a análise da utilização dos materiais partilhados no âmbito dos pré projetos de investigação, 
numa primeira fase, e nas teses, numa fase posterior, caso se consiga manter, como pretendemos, o grupo em funcionamento.

Numa fase posterior seria, igualmente, interessante alargar esta experiência a outros seminários e operacionalizar a partilha entre docentes e doutorandos através de uma comunidade de práticas online, por exemplo com as diversas unidades curriculares e seminários do curso de doutoramento em Ciências da Educação da Universidade de Trás os Montes e Alto Douro (UTAD).

\section{Referências}

AMADO, João (2103). (coord). Manual de Investigação Qualitativa em Educação. Coimbra: Imprensa da Universidade.

BARROS, Daniela (2009). "Estilos de Utilização do Espaço Virtual: Como se Aprende e se Ensina no Virtual". In Inter-Ação: Ver. Fac. Edu.UFG, 34(1):51, 74 .

DIAS, Paulo (2008). «Da e-moderação à mediação colaborativa nas comunidades de aprendizagem». IN Educação, Formação \& Tecnologias, vol. 1:4,10. disponível em http:// eft. educom.pt, consulta efetuada em 20 de março 2013, às $22 \mathrm{~h}$.

ESCOLA, Joaquim (2013). "Ciberespaço, Globalização e Interculturalidade". In CARVALHO, A. (org). Interculturalidade, Educação e Encontro de Pessoas e Povos. Porto: edições Afrontamento pp. 155,166.

GARRISON, Randy ANDERSON Terry \& ARCHER, Walter (2000). "Critical Inquiry in a Text-Based Environment: Computer Conferencing in Higher Education». In The Internet and Higher Education (2(2-3): 87-105, disponível em:

http://pepper2.ois e.utoronto.ca/ jhewitt/pepper/Uploa dedFiles/72/3/1413/COIreview.pd consulta efetuada em 2 abril 2013, às 10h.

GUNAWARDENA, Charlotte; LOWE, C. e ANDERSON, T. (1997). "Analys is of a Global Online Debate and the Development of an Interaction Analysis Model for Examining Social Construction of Knowledge in Computer Conferencing». In Journal of Educational Computing Research 17(4): 395-429, parcialmente disponível em: http://books .google.pt/books? id=Xx94veqRwLsC\&pg $=$ PA48\&lpg $=$ PA 48\&dq=GUNAW ARDENA, + C.; $+\mathrm{L}$ OWE,+ C. + e + A NDERSON,+ T. $+(1997) .+\%$ C $2 \%$ ABA nalys is + of $+a+$ Global + Online + Debate + and + the + Deve lopment + of + an + Intera, consulta efetuada a 22 outubro 2014, às $23 \mathrm{~h} 15 \mathrm{~m}$

HENRI, France (1992). «Computer Conferencing and Content Analysis». In Collaborative Learning through Computer Conferencing: The Najaden Papers. New York Springer.

LENCASTRE, José (2012). "Metodologia para o Desenvolvimento de Ambientes Virtuais de Aprendizagem: Development Research". In MONTEIRO, Angélica, MOREIRA, J. António \& ALMEIDA, Ana, Educação Online. Pedagogia e Aprendizagem em Plataformas Digitais. Santo Tirso: De Facto Editores.

Marshall e Eric McLuhan (1988). Laws of Media: The New Science. Toronto: University of Toronto.

MINHOTO, Paula, MEIRINHOS, Manuel (2011). As redes sociais na promoção da aprendizagem colaborativa: um estudo no ensino secundário. Educação, Formação \& Tecnologias, 4(2), 25-34 [Online], disponível a partir de http://eft.educom.pt

MURPHY, Elizabeth (2004). "Recognizing and Promoting Collaboration" in an Online Asynchronous Discussion". In British Journal of Educational Technology, 35 (4) :421,431.

OKADA, Alexandra (Org., 2013). Recursos educacionais abertos e redes sociais. São Luis: EDUEMA.

PINTO, Miranda (2012). "Modelo de Análise de Interações para Comunidades de Prática Online”. In Revista Ibero-Americana de Educação, nº 64:63,72.

RODRIGUES, Elvira, GONÇALVES, Daniela (2014). "Modelos de Aprendizagem Construtivistas em Plataformas Digitais". In Escola, Joaquim et al (coord). Rumo à Inclusão Educacional e Integração das TIC na Sala de Aula. Santiago de Compostela: andavira: 710-720.

WENGER, Etienne (1998). Communities of Practice Learning, Meaning and Identity. Cambridge: Cambridge University Press. 